\title{
El CUERPO SUBORDINADO Y POLITIZADO: \\ reflexión crítica sobre género y antropología médica
}

\author{
PATRICIA TOVAR \\ INVESTIGADORA DEL ICANH \\ ptovar@mincultura.gov.co
}

\begin{abstract}
Resumen
- lartículo evalúa el impacto social de la medicina en los cuerpos de las - mujeres y construye un panorama sobre la sal ud tan cercano como sea posible a la realidad social. Muestra cómo los desarroll os de la medicina afectan a las mujeres de forma diversa, lo cual apenas comienzan a tener en cuenta quienes investigan, practican la medicina o diseñan las políticas públicas. Contiene un recuento de los estudios realizados desde la antropología médica en Colombia y cómo han visto el género y la reproducción. Continúa con una crítica de la medicina, no cómo una historia de éxito que nos llevará a la cura de todas las enfermedades, sino desde su lado oscuro, que en vez de beneficiar afecta a las mujeres de manera negativa. Se refiere, específicamente, al caso de la medicina cosmética con ánimo de lucro. El análisis termina con una mirada a la violencia, doméstica y social, y a su impacto en la sal ud pública.
\end{abstract}

Palabras Clave: género, salud reproductiva, violencia, medicina, belleza.

\begin{abstract}
T HIS ARTICLE TRIES TO ASSESS THE SOCIAL IMPACT OF MEDICINE ON WOMEN'S BODIES. It attempts to construct a picture of health care as close as possible to the social reality in which we live. We will see how women are differently affected by medical developments; differences that have only recently been taken into account by researchers, practitioners, and policy makers. It reviews medical anthropology studies carried out in Colombia, examining how they have looked at gender and reproductive issues. It continues with a critique of medicine, seeing it not as a success story that will provide cures to all diseases, but its dark side than affects women's health in a negative way. I specifically make reference to the case of for profit, cosmetic medicine. I close this analysis with a look at domestic and social violence and its impact on public health.
\end{abstract}

KEY WORDS: Gender, reproductive health, body, violence, medicine, beauty.

\author{
Revista Colombiana de Antropología \\ Volumen 40 , enero-diciembre 2004 , pp. 253282
}




\section{NTRODUCCIÓN}

5 N EL AMPLIO ABANICO DE LAS PROBLEMÁTICAS ESTUDIADAS POR LA antropología médica en Colombia, es posible ver el aumento de trabajos que abordan la sal ud con perspectiva de género*, y, dentro de éstos, los estudios sobre mujeres y salud. Sin embargo, excepto por la atención exclusiva y a veces esencializantequese presta a la reproducción,

* Agradezco la colaboración de la antropóloga Mónica Eliana Velasco en la búsqueda de documentación para la elaboración de este artículo. son pocos los trabajos con una mi rada crítica o queliguen las diferentes real idades que se inscriben en el cuerpo de las mujeres.

El reconocimiento dela importancia dela categoría "salud dela mujer" es reciente y se debe a iniciativas y priori dades de entidades como las Naciones Unidas, que pasaron del interés general centrado en el control de la población, propio de los años 1950 y 1960, a un nuevo marco conceptual y teórico enmarcado en los derechos humanos, la creación de mejores condiciones de vida para los grupos en desventaja y la promoción de las mujeres como actoras y gestoras con capacidad de decidir sobre sus cuerpos.

En el ámbito internacional, muchos aspectos han recibido atención en relación con el género y la salud, como la crítica femi nista de la ciencia, las enfermedades detransmisión sexual, el VIH/sida, el cáncer de seno y el de cuello uterino, Ia nutrición, la anorexia, Ias manipulaciones quirúrgicas del cuerpo, los diferentes tipos de violencia y su efecto sobre la salud de las mujeres y los aspectos rel aci onados con la sal ud ocupacional. Dentro del análisis dela reproducción seresal tan lamenstruación, el parto, Ia lactancia y la menopausia, el control de la natalidad, el aborto y el impacto de las nuevas tecnologías reproductivas.

Dentro de la antropología médica confluye una amplia gama de perspectivas teóricas y metodológicas, al gunas de ellas reñidas entre sí. Esta reflexión parte del cuestionamiento de la visión humanitaria dela medicina y la salud pública, que no tiene en cuenta cómo los intereses capital istas, sexistas y racistas influyen en la situación de salud y redefinen los propósitos de los servicios de salud. En este caso, me refiero a la biomedicina, es decir, al sistema médico dominante, basado en el conocimiento científico occidental que se imparte en universidades y se ratifica 
en medios académicos. Este sistema ha sido criticado, entre otras razones, por centrarse en los aspectos biológicos, sin casi tener en cuenta los sociales, especializándose en un solo órgano o parte del cuerpo y olvidándose de la persona afectada. Existen también algunos eventos en el desarrollo de la medicina y la salud pública del sigl o veinte que han afectado directamente a las mujeres, mal tratando incluso sus derechos humanos, como el control poblacional forzado, que reflejan intereses políticosy económi cos global es basados en las desi gual dades sociales.

La antropología médica analiza también aspectos como la organización social, el género, el parentesco, los sistemas de matrimonio, la etnia, la clase social y la religión, y su contribución al estudio del bienestar y las desigual dades en la salud. Es claro que las mujeres se enfrentan a ciertas condiciones que son exclusivas o más notorias en ellas y que tienden a ser ignoradas (Doyal, 1995). La salud, como sabemos, está profundamente ligada al contexto familiar, comunal y social, al grupo étnico y al estrato social; de ahí la importancia de di señar programas apropiados a las necesidades de cada grupo. El género cumple un papel muy importante en la posibilidad de tener una vida saludable, de sufrir ciertas enfermedades, de sobrevivir a los primeros años de vida o dellegar a la vejez. También hay condiciones específicas quellevan a patrones de enfermedad diferenciados, como en el caso de las afecciones coronarias o los traumas de la violencia social, que afectan más a los hombres; o las complicaciones del parto, que hasta hace medio siglo eran la principal causa de muerte en las mujeres.

La modernización de la salud trajo consigo una revolución demográfica que cambió los patrones de mortali idad y aumentó la esperanza de vida de las mujeres. Gl obal mente, esto ha modificado aspectos como las percepciones sobre el ciclo de vida, lo que tiene también gran impacto en la organización de los sistemas de salud y seguridad social, que repercuten en el dilema del cuidado institucional de los ancianos frente al cuidado en la casa, hecho por las mujeres sin remuneración al guna. Esto afecta la idea de vejez, las relaciones entre generaciones, la naturaleza de la vida familiar, la estructura de las comunidades y el presupuesto de las naciones.

En la historia de la antropología médica en Colombia abundan los materiales sobre chamanismo, curanderismo, plantas medicinales, enfermedades tropicales, mal de ojo y medicina 
tradicional, los que, por lo general, no cuentan con enfoque de género y han ignorado el papel de la mujer como chamana, curandera, conocedora de plantas, es decir, como agente en el proceso de sanar, con conocimientos y experiencias diferentes a las de los hombres. Tampoco se le ha prestado atención suficiente como usuaria de los servicios médicos, no se ha examinado su papel como trabajadora de la salud -en su desempeño como enfermera, auxiliar o médica- ni el impacto quetienen en ellas las condiciones económicas, políticas y cultural es, en particular la legislación reciente sobre salud.

En nuestro contexto es posi ble encontrar, entonces, diversos enfoques relativos a la salud y el género poco tratados, a los queharéreferencia en este artículo. Comenzaré por examinar la ciencia y la medicina desde los trabajos de la crítica feminista, con el fin de ver las conexiones entre los roles de género y la ideol ogía, perpetuados por el lenguaje científico, las construcciones culturales, la medicalización del ciclo de vida y el impacto de las nuevas tecnologías en la salud, elementos que se entrecruzan, a su vez, con las políticas públicas y la producción social de la salud, lo que en este caso nos liga de nuevo con la salud sexual y reproductiva.

Exami naré luego las ideologías, manipulaciones y violencias que hay sobreel cuerpo femenino como respuesta dela medicina a la moda, y la imposición de imágenes y sus efectos sobre la salud, como en el caso del abuso de cirugías estéticas y sus consecuencias nefastas; y, rel acionado con lo anterior, los problemas de desnutrición como la anorexia, para pasar al impacto de la violencia en la salud. En el caso del conflicto armado se reportan problemas diferentes asociados para cada género, como el abuso sexual en el caso de las mujeres y las lesiones corporales en el de los hombres. En las zonas de confrontación surgen afecciones comunes en hombres y mujeres, como, por ejemplo, el estrés postraumático.

Pongo sobre la mesa estos aspectos con la intención de brindar elementos para una discusión más profunda, quesi rva deguía de investigación sobre la dimensión de género y su intersección con la salud, la cultura, los grupos étnicos y la clase social, Ilamando la atención sobre los efectos deletéreos del sexismo, el racismo y la pobreza. Se trata de ir más al lá de describir cómo se practica la medicina en zonas urbanas o en los diferentes grupos étnicos, y de mostrar las creencias que existen al rededor de la 
salud, para examinar de qué manera se construyeel conocimiento médico, el impacto de la medicina con ánimo de lucro y los dilemas éticos que se entrecruzan con las políticas estatales y la producción social dela salud.

\section{LA CONSTRUCCIÓN DEL CONOCIMIENTO MÉDICO Y LA CRÍTICA FEMINISTA \\ DE LA CIENCIA}

ARTO DE LA IDEA DE QUE LOS CONCEPTOS DE SALUd Y ENFERMEDAD son históricos y han sido claramente diferenciados según el género; más aún, están conectados a procesos políticos y hacen parte de la cultura y la manera como ésta enmarca las relaciones social es. Las mi radas críticas a la medicina han resal tado la retórica sobre las metáforas masculinas y mi litaristas características del lenguaje de la ciencia, en el que proliferan exageradamente térmi nos como "invasión, ataque, enemigos, agresión, y defensas", por nombrar sól o al gunos. Susan Sontag (1989) habló del lenguaje de la paranoia política y de las metáforas utilizadas para describir las enfermedades, y de cómo contribuyen éstas a estigmatizar y aterrorizar a quienes las padecen, como en el caso de la lepra, el cáncer y, más recientemente, el sida.

Por otra parte, el lenguaje de la ciencia y la medicina se discute desde un marco sexista, en el que lo femenino se asocia a Io natural, al mundo de las emociones y del cuerpo, y es visto, por tanto, como inferior a lo masculino, que, además, asume su control (Haraway, 1991; Merchant, 1989). Una de las contribuciones de estas críticas es el hecho de que la ciencia y la medicina ya no son consideradas como si estuvieran libres de prejuicios, no tuvieran una fuerte carga cultural y fueran ajenas a los juicios de valor, ni tuvieran el peso de los significados culturales. En otras palabras, el mito de la "neutralidad" dela ciencia se ha rebatido.

El lenguaje, como se sabe, moldea la percepción de la realidad, afecta nuestras reacciones y sirve de puente entre la sociedad y el individuo. Con esta idea en mente, Martin (1987, 1990) ha trabajado la epistemología y el lenguaje de la biomedicina y ha criticado la manera negativa en que los textos de medicina representan el cuerpo y los procesos biológi cos femeninos. Muestra, 
por ejemplo, de qué manera en la visión del cuerpo como máquina se enfatizan aspectos como el control, las jerarquías y la producción. Términos tan comunes como el "trabajo de parto" implican eficiencia y, por oposición, la menstruación y la menopausia se ven como fallas y deficiencias. Los procesos masculinos, entre tanto, se describen como grandiosos, activos y maravillosos (Martin, 1996). Todo esto ha permeado el lenguaje cotidiano. Así, por ejemplo, una revista muy vendida describe Ias experiencias de un donante de semen en Profamilia: “Armado con un frasco parecido al delas muestras de orina, pero destinado a depositar el brioso material genético, entro y cierro la puerta (énfasis mío)"1.

Los avances de la medicina que permitieron comprender el proceso de la concepción fueron lentos y reflejan también la posición de la mujer en la socie-

1 Artículo publicado en la revista Aló. 419 .30 de noviembre-9de diciembre de 2004. Bogotá. dad. Así, por ejemplo, es considerada como la parte pasiva de la reproducción (Uribe, 2002). Durante muchos siglos se creyó que el hombre contribuía con el el emento más importante para la formación del ser humano. Los de ella eran, apenas, un aporte, informe y sangrante, que se expulsa mensualmente cuando no hay fecundación, frente al del varón, que contribuye con la semilla, donde se creía radicaban las características humanas más esenciales. Según Martin (1996), en lo textos médicos la concepción se presenta no como un evento en el que interactúan mutuamente un óvulo y un espermatozoide, sino como la conquista por parte de un agente activo y agresivo, que penetra, perfora y lucha después de una competencia y un peli groso recorrido hasta "invadi r" o "col onizar" una célula que está esperando ese momento, pasivamente.

Se ha escrito bastante sobre la medicalización y comercial ización de los procesos biológicos de la mujer, como en el caso de la controvertida terapia de reemplazo hormonal (TRH), anunciada como el remedio apropiado que nos ofrece la ciencia moderna para "curar" el problema de la menopausia. La industria farmacéutica se ha lucrado enormemente y ha desempeñado un papel importante en infundir ideas y crear necesidades en las mujeres sobre el uso de hormonas desde la pubertad hasta la vejez. Tanto las pastillas anticonceptivas como la TRH se mercadean con la ilusión de la belleza y de la eterna juventud, ocultando en letra di mi nuta los riesgos col ateral es - sobre los cual es 
hay bastante discusión- y enfatizando propiedades para las que no fueron diseñadas. La menopausia es vista como un problema de deterioro, prácticamente como una enfermedad, que requiere de terapia, por lo que la TRH se promociona como la panacea para la osteoporosis, los problemas del corazón y, en general, los efectos "desagradables" dela vejez. Sin duda, es posi ble hablar de explotación de las mujeres por parte de la industria farmacéutica cuando sepromocionaun examen casero, como el dela prueba de embarazo, para determinar si ya se ha entrado en la menopausia ${ }^{2}$. Todo esto dentro de un contexto cultural en el cual decirle a una mujer "menopáusica" es un insulto.

Recordemos que la industria farmacéutica esconde capítul os oscuros y vergonzosos como el de la talidomida, medicamento que se recetó sin discriminación a mujeres embarazadas con la idea de evitar la náusea y el mareo matutino, y produjo bebés con malformaciones. A pesar de esta tragedia, esa droga se está experimentando para curar complicaciones de la lepra-eritema nudoso leproso- y de ulceraciones graves asociadas al sida ${ }^{3}$. Aun cuando entidades como la Food and DrugAdministration de Estados Unidos ejercen control, es pre-

2. http:// www.timegoesby. net/ 2004/ 04/ the_menopause_i.html

3 Véase la página web de la organización March of Dimes. http:// www.nacersano.org/ centro/ 9388_9939.asp ocupante que se comercialice de nuevo sin garantizar los cuidados extremos necesarios.

La medicina ha representado a las mujeres de maneras diferentes según el momento histórico. Así, como dice Uribe (2002), la ginecoobstetricia aparece en el siglo dieciocho, cuando "se inventa la mujer" como un ser con derechos, con un cuerpo, parte del "binomio indispensable parala reproducción humana". Antes se la veía como un hombre defectuoso e incompl eto, y sus órganos reproductivos como correspondientes, anál ogos o complementarios a los del hombre (Schiebinger, 2004). Con los avances en el estudio de la anatomía se encontró que el útero no tenía nada análogo en el cuerpo masculino, pero no por eso ha dejado de ser cal umniado, descrito como una animal, como lo hizo Platón en la antigüedad, o responsabilizado por problemas psicológicos como la histeria. Entre sus muchas representaciones, aún se describe en li bros de medi cina como un órgano análogo a las frutas, por ejemplo, del tamaño de una pera; los ovarios se muestran como ciruelas o al mendras y los médicos advierten que no sirven 
para nada, excepto para producir miomas o para su función primordial, la reproducción (Tovar, 2004).

Lo anterior nos lleva a plantear la discusión existente sobre el uso de tecnologías en el proceso reproductivo como la ecografía y la amniocentesis, procedimientos prenatales rutinarios en muchos países (Rapp, 1990). La ecografía da a la experiencia del embarazo una dimensión diferente de pertenencia y de relación con el feto, al permitir observar su desarrollo y, sobre todo, su sexo. Aun cuando útil para detectar complicaciones, este, como otros procedimi entos, contribuye a crear jerarquías y distancias que refuerzan la autoridad del médico basada en la tecnología y disminuyen la importancia del conocimiento de la mujer sobre su propio cuerpo (Sargent y Brettell, 1996). Por otra parte, la amniocentesis, un procedimiento riesgoso de análisis del líquido amniótico, permite detectar anomal ías genéticas graves como el síndrome de Down, lo que daría a la madre el conocimiento suficiente para tomar la decisión de practicarse un aborto terapéutico. Obviamente, esta tecnología no ha tenido impacto en un país como Col ombia, donde el aborto no es legal, pero en otros como India y China tiene efectos perversos, pues el interés primordial dela prueba es abortar el feto si es de sexo femenino, lo que ya ha producido desbalances demográficos (Browner, 1995, Hubbard, 1985).

En cambio, otras tecnologías de fertilidad sí han Ilegado al país, como puedeconstatarseen la prensa, donde, devez en cuando, aparecen solicitudes de "alquiler de úteros", con ofertas de compensación económica para la joven, educada y de buena presentación personal, que se requiere para llevar a término un embarazo a partir de la implantación de un embrión que genéticamente pertenece a otras personas. En el país ya nació la primera bebé que se formó a partir de un óvul o congelado. Las posi bi lidades sehan abierto y asuntos como el delas células madre se discuten en el congreso de la república. El folleto de promoción de la Compañía Record de Colombia, que maneja el banco nacional de células madre dice: "Al gún día [las células que están dentro de la placenta y el cordón umbilical de su bebé] podrán salvar la vida de su hijo o la de otro miembro de su familia". Y, por un pequeño "costo/beneficio, la inversión es muy accequible (sic)". Un uso más para la placenta humana que cruzó de otra manera la línea de lo simbólico a lo lucrativo, lejos ya de sus connotaciones rituales, y quesale de las mujeres y regresa a el las 
también en forma de costosos productos para la belleza, para reducir el proceso natural de envejecimiento dela piel, para tratar heridas y quemaduras y para fortalecer el sistema inmunológico ${ }^{4}$.

Nuestra tarea es, por tanto, proponer al ternativas de conocimi ento con cami nos epistemológi cos y categorías científicas diferentes, que tomen en cuenta el anál isis del poder, incluyan la crítica de la dominación, explotación y exclusión de las mujeres y el monopolio masculino de la estructura social y la producción y práctica del conocimiento científico. Esta propuesta permite identificar los sesgos que aparecen en el estudio de la salud y la enfermedad, la práctica médica o la respuesta social a la enfermedad y el saber médico. Es decir, cómo se percibe y define el bienestar, que en el caso de las mujeres se complica, como veremos más adelante, cuando entran en juego las categorías de belleza y edad.

La crítica de la ciencia muestra los sesgos de género en la literatura científica, que se asume como objetiva y con validez universal. Por ejemplo, existela tendencia a consi derar a la mujer como "más frágil", a pensar que la afectan más las tensiones emocionales mensuales y diarias, que percibe el dolor de diferente manera e, incluso, que sufre de inferioridades, no comprobadas, de orden biológico; es decir, se asume que la composición genética de las mujeres y los hombres es distinta. De ahí surgen varios cuestionamientos: ¿hasta qué punto estos supuestos afectan la calidad de los servicios médicos reci bi dos por las mujeres? Dentro del análisis de las dinámicas de poder se ha cuestionado también la interacción entre la persona que practica la medicina y la queconsulta. ¿Cuántas mujeres dejan de ir a una consulta gi necológica porque les afecta quelas examine un hombre? ¿Ocurre lo mismo a los hombres cuando los examina una uróloga, en los pocos casos en que aparezca al guna, pues son muy es-

4. http://www. milaskincare.com/timeline.html. Se encontraron más de quince mil sitios web sobre placentas humanas y belleza

5 De acuerdo con la Sociedad Colombiana de Urología, en 2005había cuatrocientos treinta y seis especialistas registrados, de los cuales cuatrocientos treinta y uno eran hombres y cinco mujeres; es decir, ellas sólo representaban $1,1 \%$. casas las que escogen esa especialidad?5. ¿Qué pasa cuando el pediatra es un hombre y regaña, desautoriza o cul pabiliza a la mamá del ante de sus pacientes? 


\section{LA ANTROPOLOGÍA DE LAS ETAPAS DE LA REPRODUCCIÓN}

A REPRODUCCIÓN HA SIDO LA BANDERA DE LOS MOVIMIENTOS FEMInistas del sigl o vei nte, encontrándose, por una parte, la lucha por el derecho a deci dir cuándo y cuántos hijos desean tenerse y, por otra, cómo quisieran tenerse (Petchesky, 1998). En los medios académicos colombianos, los diversos momentos de la reproducción -menstruación, fecundidad, parto, menopausia, aborto- y el crecimiento humanos -lactancia y nutrición- empezaron a recibir atención tardía, l uego de que en algunos otros países latinoamericanos ya hubiera estudios históricos al respecto, como los de Cosminsky (1977) y Vargas y Matos (1973) sobre la partería indígena.

Estudios más recientes continúan examinando los saberes populares sobre el parto, así como conocimi entos sobre las prácticas abortivas, Ias relaciones de género en la pareja, Ias enfermedades detransmisión sexual y la violencia sexual en un marco de construcción de identidad cultural (Bailey et al., 1988; Leal Garza et al., 1995; Li, 1992; Viveros, 1997; Wartenberg y Salcedo, 1997; Zamudio et al., 1992). Se ha trabajado, además, sobre las posibilidades económicas y la disminución en la cobertura de Ios servicios de salud apoyados por el estado (Tabares, 1994), y acerca del embarazo, el parto y la lactancia, como en el caso de la mujer ingana (Guevara, 1997).

Por otra parte, muchos trabajos sobre salud sexual y reproductiva reseñan críticamente los diferentes factores que determinan la salud de la mujer no indígena, urbana o campesina. Dentro de esta área se examinan aspectos como el crecimiento o disminución de la población, cambios en los patrones matrimoniales, el tamaño de la familia, los métodos de control natal, el embarazo juvenil (Rico de Alonso, 1986), I os procesos de decisión masculinos (Viveros y Gómez, 1998) y el aborto (Wartenberg, 1999). Como parte de este tipo de anál isis caben también la situación general de las mujeres y sus derechos en cuanto a la educación y al trabajo, los cambios en el estilo de vida y las creencias religiosas.

El estudio de Viveros y Gómez (1998) sobre la salud reproductiva incorpora, por fin, la perspectiva masculina, poco tenida en cuenta, pues se asume que la reproducción es asunto femenino. 
Las masculinidades en grupos étnicos también han sido consideradas importantes, como lo ilustra el estudio de Urrea et al. (2004) acerca de grupos afro en Cali.

\section{La menstruación}

A ANTROPOLOGÍA HA EXAMINADO LA MENSTRUACIÓN, SOBRE TODO, DESde el punto de vista simbólico (Buckley y Gottlieb, 1988) y de I os tabús asociados a ella (Douglas, 1977; López, 1945). No obstante, ésta ha sido muy poco estudiada en Colombia. Aunque hay referencia a ritual es femeninos de pubertad casi desaparecidos ahora, como la majayura en La Guajira, el ritual de "la pelazón" en el A mazonas y el suruwa, documentado por Ortiz y Pradilla (1987) en Ios Ilanos oriental es. Gálvez et al. (2002), por su parte, muestran el papel del ritual de iniciación para socializar a la joven en sus labores de adulta ${ }^{6}$-al recibir consejos y conocimientos de las mujeres mayores-. Analizan también datos sobre la edad de la primera menstruación en niñas de poblaciones indígenas, en algunos casos, en promedio, a los once años.

6. Es necesario recalcar que mientras existen numerosas y extensas descripciones sobre los rituales masculinos de iniciación, las relacionadas con las niñas son mucho menos.

Por otra parte, en medios urbanos se habla de aspectos como el síndrome premenstrual (Davis, 1996), sosteniéndose que la cultura y la política afectan y determinan la menstruación, el parto y la menopausia, y que cada vez están más medicalizados.

\section{El parto}

A PARTiR de la déCAda de 1980 comenzó a examinarse el parto en A comunidades indígenas, resal tándose los aspectos cultural es que lo rodean dentro de un sistema "etnomédico" de creencias, prohibiciones y prescripciones en la dieta de la gestante y la descripción del ciclo vital (Mckee, 1982; Gutiérrez de Pineda, 1985 Alcaraz, 1993 Gutiérrez y López, 1999).

Es importante referirse también al control sobre el proceso del parto y a la crítica hecha a la manera como se lleva a cabo 
dentro de la medicina institucional, en la que casi siempre son hombres quienes lo atienden, en camillas rígidas e incómodas, utilizando arneses, en un proceso hospitalario despersonalizado, sin que los padres se involucren. Por otra parte se cuestiona la al ta tasa de cesáreas, la ci rugía que más se hace a las mujeres, y la frecuencia con que se real izan histerectomías (Tovar, 2004).

\section{La fecundidad y la reproducción}

E N ESTE CAMPO, Y RELACIONADO CON LOS SABERES POPULARES, ES DE destacar el estudio ya mencionado, de Gálvez et al. (2002), sobre la vivencia de la fecundidad entrelas mujeres indígenas del departamento de Antioquia. En este trabajo se reporta la coexistencia de métodos tradicional es basados en la botánica regional con el acceso y apropiación de anticonceptivos occidentales, en un marco de presión en el cual la fecundidad se valora y la anticoncepción se sanciona como una agresión más a grupos que tienen amenazada su continuidad, por lo queen caso detransgresiones existen tensiones y se castiga a las mujeres con el encierro o la privación de la libertad. La fecundidad, se concluye, es de gran importancia en el sistema indígena de prestigio, en el que prima la maternidad y sus funciones derivadas de tareas en el ámbito doméstico. Esta investigación resalta los di lemas en relación con el cuerpo y la fecundidad en las comunidades indígenas, donde existen altos porcentajes de mujeres multigrávidas con problemas de salud reproductiva y con pocas opciones.

En cuanto a la reproducción en relación con la fecundidad, cabe anotar que ha si do más estudiada en zonas urbanas (Arango, 1992; Puyana, 1985) y desde el punto de vista de la relación médico-paciente (Ramírez, 1983).

\section{Lalactancia y la nutrición}

A Un CUANDO LA LACTANCIA TAMBIÉN HA RECIBIDO POCA ATENCIÓN DE
nes hechas en el país muestran que la salud y la cal idad de vida
delas mujeres indígenas son, por lo general, desoladoras, y aun- 
que describen los principal es problemas de sal ud, las propuestas se centran en la cultura y la utilización de una al imentación más bal anceada. Existen, por fortuna, críticas a las deficiencias del sistema de salud nacional, negligente frente a la precaria situación de la salud en los grupos étnicos minoritarios (Castañeda, Billings y Blanco, 2003 Alcaraz 1993).

En el estudio publicado por Gálvez et al. se incluyen los periodos de lactancia, Ias demandas nutricional es, la ingestión de alimentos, la actividad física y el uso de lactógenos entre las mujeres indígenas del departamento de Antioquia. Desde una perspectiva gl obal, Baumslagy Michels (1995) cuestionan alas compañías fabricantes de leches para bebés, con énfasis en las políticas que se mueven en esta industria.

Otro aspecto de la salud relacionado con la maternidad es la nutrición y sus aspectos culturales y políticos. Se sabe quecuando hay hambrunas quienes mueren primero son los bebés, luego las mujeres y, final mente, los hombres, pues en muchas culturas tienen el privilegio de comer primero y mejor que el resto de los integrantes de la familia (Lindenbaum, 1979, Merchant y Kurz, 1993). Esta cuestión de la comida y el hambre nos remite a los costos y los beneficios de tener hijos, lo que debe verse en el marco de indicadores de mortalidad y morbilidad infantil y su relación con la salud dela madrey su situación económica (SchaIler y Nightingale, 1992).

Gálvez se refiere también a “I os control es demográficos ocultos", mediante los cuales, por ejemplo, el suministro de al imentos a los ancianos se reduce, y se "elimina" una parte de la población infantil cuando el jaibaná diagnostica una enfermedad para la que no hay remedio. Observa también cómo se presta menos atención a los niños con malformaciones y cómo en el caso de un parto múltiple uno de los bebés reci be menos cuidados, con lo que sus posibilidades de fallecer se incrementan (Hernández, 1997, citado en Gálvez et al., 2002), situación que examina Scheper-Hughes (2002) en el contexto de la pobreza en el Brasil. Otra práctica es la dela destrucción de género indeseado, por lo general alas recién nacidas, hecho que se considera un grave problema en China e India (Miller, 1997). 


\section{El aborto}

E l aborto en Colombia es un ASUnto muy Sensible, y SU CARÁcter ilegal y quela igl esia católica-que aún tiene gran influenciase oponga a su práctica no si gnifica que no exista; por el contrario, su ilegalidad y clandestinidad -que suponen condiciones inseguras- Ilevan a índices de morbilidad y mortalidad femenina muy elevados, que afectan en proporción considerable a las jóvenes. Según el estudio de Zamudio, Rubiano y Wartenberg (1992), el aborto inducido y sus complicaciones continúa siendo la segunda causa de mortali dad materna en el país, con 16\%. Esta investigación muestra también que $26,5 \%$ de las mujeres que que dan embarazadas son menores de veinte años; de éstas, casi la mitad (44,6\%) se ha practicado un aborto. En consecuencia, existe un problema muy grave de salud pública, del que se sabe su existencia pero que no se asume con la atención requerida.

Según un informe de la Presidencia de la República y del Ministerio de la Protección Social, basado en datos del Instituto Nacional de Medicina Legal y Ciencias Forenses, el alto porcentaje de muertes por complicaciones del aborto es preocupante. A lo anterior se añade que poco más de la mitad de los embarazos en Colombia no son deseados ${ }^{7}$. No obstante, para reducir $50 \%$ la mortalidad materna se puso en marcha un "plan de cho-

7. http:// www. presidencia.gov.co/sne/2004/ agosto/ 25 17252004.ahtm,

que", pero el gobierno nacional no considera la posibilidad de despenalizar el aborto como una posibilidad de enfrentar este problema. En el informe citado, la directora desal ud pública del Ministerio dela Protección Social indicó que, además, una de las situaciones más preocupantes es que en el país están empezando a morir mujeres con edades de entre diez y catorce años por causa de la interrupción del embarazo.

El trabajo deViveros (1997) examina el aborto desde una perspectiva sociocultural y hace un seguimiento de dos décadas; ella sugiere tratarlo como una cuestión política y, a la vez, religiosa, pues resal ta los discursos "antiaborto" de grupos conservadores y la oposición de la iglesia católica a los intentos de modificar la legislación y de plantear excepciones a su prohibición. Según esta investigadora, el problema poco tiene que ver con el aborto en sí mismo, sino que es, más bien, un pretexto 
para expresar distintas formas de articular lo biológico, lo político y lo social. Es decir, se refiere a otros aspectos fundamentales en la organización social como son las desigual dades en el campo de las relaciones entre hombres y mujeres, la familia, la maternidad y la responsabilidad individual y colectiva. Hace referencia, además, a la doble moral y a las contradicciones que existen entre la amplia oferta de este servicio en las grandes ciudades, incluso en condi ciones higiéni cas, lo queayuda en cierta manera a disminuir la mortalidad femenina pero permite multiplicar los establecimientos que practican abortos inescrupulosamente, anunciando demanera abierta sus servicios en la prensa. Viveros muestra cómo la doctrina católica humaniza el feto y deshumaniza a la mujer, quien termina siendo considerada, apenas, como el "receptáculo" o la "incubadora" de otra vida. Por otra parte, no está sobre la mesa la discusión de la violación, el incesto y otros crímenes dirigi dos hacia las mujeres, que resultan en embarazos no deseados.

Desafortunadamente, el género no se asume como una parte integral delas agendas de investigación ni delas políticas públicas, no sólo en salud, sino, en general, pues se supone que en Colombia a partir de la constitución política de 1991 las políticas públicas incluyen mujeres y hombres en condición de igualdad (García, 2003).

\section{La menopausia}

P

ARA TERMINAR, LA REFLEXIÓN SOBRE LAS MUJERES Y LA REPRODUCCIÓN no puede dejar de lado la menopausia. El estudio de este proceso en comuni dades indígenas o en áreas urbanas no forma parte todavía en las prioridades de la investigación dela antropol ogía médica en Colombia, excepto por menciones pasajeras. Podría argumentarse al respecto quela mayor parte de los trabajos sobre la reproducción son hechos por mujeres, quienes observan y comparan desdesu propia condición de sal ud y edad, pero es posible sostener también que se debe a la carga tan negativa que tiene en nuestra cultura, la cual la hace algo que no se menciona, al oponerse a la sublimación de la maternidad. No se han reportado ritual es de transición a esta etapa de la vida ni sus aspectos positivos relacionados con la li beración de los riesgos y las cargas de 
la maternidad, aun cuando sí se ha hablado del papel positivo que asumen las mujeres mayores en los aspectos públicos de la comunidad, que les estaban vedados previamente (Davis, 1996).

\section{LA RELACIÓN ANÓMALA \\ ENTRE LA MEDICINA Y LA BELLEZA}

AL Y COMO SE PRESENTA EN LA ACTUALIDAD, EL ÉNFASIS EXAGERADO EN la belleza femenina y la manipulación del cuerpo tiene repercusiones psicológi cas, físicas y políticas en la salud personal y pública. Resulta, además, en opresión, control y disminución del empoderamiento, esclavizando a la mujer y encasillándola en un rol artificial. Esto es, siguiendo a Foucault, una manera de disciplinar el cuerpo hasta tornarlo "dócil" y subordinado.

La presión que existe para obtener un ideal de belleza -que cambia constantemente-, ha resultado en altos costos físicos, psi cológi cos y económicos para la salud de las mujeres. Los efectos psicológicos relacionados con la búsqueda del cuerpo "perfecto" son bien conocidos, pues además de los casos extremos de la anorexia y la bul imia hay comportamientos obsesivos, confusión, inseguridad, baja autoestima e infel icidad. Hombres y mujeres manejan de manera diferente el ejercicio como una manera de mantenerse en forma: ellos lo hacen para aumentar su masa muscular y fortal ecer el sistema cardiovascular, mientras queellas están interesadas en perder peso y mol dear al guna partedel cuerpo para aumentar sus atractivos (Sal tzberg y Chrisler, 1995). Los hombres consumen esteroi des para aumentar su masa muscular y las mujeres ingieren fármacos y otros productos para no sentir hambre y el iminar la grasa.

Las imposiciones de la moda para realzar alguna parte del cuerpo, como el uso de zapatos de tacón al to y con punta, producen problemas de espalda, callos y juanetes dolorosos que dificultan la movilidad. Los tacones y los trajes apretados y cortos impiden correr en caso de peligro. Las mujeres se han envenenado, han desarrollado infecciones por el uso de sustancias tóxicas, han sufrido quemaduras y laceraciones en la piel por depilarse o tratar de cambiar el color de la piel o la textura del pelo. Incluso arregl arse las uñas en un salón de belleza implica el riesgo decontraer una infección, si la higienees deficienteen 
los instrumentos usados, pues hay probabilidad decontacto con sangre.

La historia del vestido femenino y de la decoración corporal está llena de sujeción del cuerpo, acompañada de dolor y hasta de tortura. El corsé impedía respirar y caminar normal mente y era el causante de ahogos, malestares y complicaciones del embarazo. Ninguna otra parte del cuerpo ha sido aplanada, escondida, resaltada, puesta en punta, redondeada, subida, bajada, agrandada o reducida como el seno. Sobre el tema del busto es importante tener en cuenta el aporte de Elías Sevilla (2003), quien detalla su "iconografía", anal iza la atención exagerada que este recibe en la ciudad de Cali y ofrece datos etnográficos que muestran la voz de las mujeres que se someten a operaciones para cambiar el tamaño del seno.

La mani pulación del cuerpo y su decoración es una expresión muy antigua en la historia de la humanidad. El adorno y la modificación corporal, que puede ser temporal o permanente, se ha utilizado para marcar diferencias étnicas, religiosas, de rango y de género. El repertorio de prácticas es amplio e incluye las perforaciones, hoy Ilamadas piercing, la escarificación, las mutilaciones, los tatuajes, las modificaciones delos dientes, el estiramiento del cuello, las deformaciones del cráneo, el cuello, las orejas y de muchas otras partes del cuerpo. Obviamente, esto tiene implicaciones en la salud, pues hay un riesgo de infección y transmisión de enfermedades e, incluso, de muerte. No obstante, sus dimensiones e implicaciones van más allá; así, por ejemplo, los "pies de Ioto" -cómo eufemísticamente se llamó a la práctica china de fracturar y deformar los pies de las mujeres con la idea de que entre más pequeños más eróticos, lo que significaba sometersea un sufrimi ento enorme- tenía como resultado el control del cuerpo femenino restringiendo el movimiento. La infibulación ${ }^{8}$ y la clitoridectomía $a^{9}$-o circuncisión faraónica (femenina)-, realizadas en casa, son un grave problema de salud pública y de derechos humanos en muchos países de África y se relacionan directamente con las costumbres matrimoniales (Gordon, 1991). Ambos procedimientos están relaciona dosy se practican demanera ritual, en diferentes grados de severidad, con el fin de asegurar la castidad y eliminar el deseo sexual dela mujer;

8. Práctica tradicional en zonas africanas islámicas, por medio de la cual se suturan los genitales externos de las niñas, dejándoles apenas un pequeño agujero para la orina y la menstruación.

9. Consistente en la remoción o mutilación del clítoris. 
las secuelas del daño físico y de las condiciones antihigiénicas en que se practican tienen graves consecuencias.

Las sociedades modernas y urbanas no están exentas de esto y muestran muchas contradicciones al respecto. La medicina, Ia cienciay, de nuevo, la industria farmacéutica, han entrado de lleno en el negocio de la belleza. Está, por ejemplo, el caso del Botox ${ }^{\circledR}$ empleado en la "terapia" de arrugas y para "eliminar vicios de expresión". Este producto es una toxina (Clostridium botulinun) que causa el botulismo, enfermedad grave asociada a la ingestión de al imentos en mal estado, que produce parálisis del sistema nervioso y, en muchos casos, la muerte. El Botox ${ }^{\circledR}$ está aprobado en Estados Unidos sólo para su uso médico en el tratamiento de ciertos desórdenes musculares, pero no para su uso cosmético. Es tan popular que grupos de personas se reúnen en cuartos de hotel, en oficinas o en sus casas y realizan una fiesta/ritual en la que un especial ista oficia y lo inyecta en la cara a los asistentes con el fin de paralizar las zonas donde hay $\operatorname{arrugas}^{10}$. No se recomienda a mujeres embarazadas o lactantes. Igual ocurre con el Rogaine ${ }^{\circledR}$, que se vende de manera separada para hombres y mujeres, por los daños que puede producir en el feto. Estees un producto diseñado inicial mente para tratar la presión arterial al ta, pero que entre sus efectos colateral es producía la aparición de pel o corporal. Hoy en día se vende en cual quier supermercado en la sección de cosméticos, para ser aplicado sobreel cuero cabel ludo. Al parecer, los hombres son los principal es consumidores de este producto y de otros tratamientos promocionados para la calvicie, entre los que se incluyen, también, la cirugía.

Ostentando títulos de prestigiosas universidades, los médicos, casi todos hombres, imponen su estética con el bisturí11 , anunciando por todos los medios de comunicación "métodos" correctivos y "soluciones" donde se proponen

10. Véase http:// www.fda.gov/ fdac/ features/ 2002/402_botox.html. Una búsqueda por internet con la palabra Botox, mostró 2'270.000 sitios.

11 La página web de la Sociedad Colombiana de Cirugía Plástica-Estética, Maxilofacial y de la Mano (SCCP) lista entre sus ciento cincuenta y cinco miembros asociados a treinta y una mujeres, es decir, $20 \%$. Este porcentaje es más alto que el de México $(16,7 \%)$ y Argentina $(7,9 \%)$. www.cirugiaplastica.org.mx/ www.cirplastica.org.ar/ "cuerpos perfectos" y se ofrecen "milagros", principalmente para las mujeres, para lo que se muestran fotografías de "antes" y "después" y se vende la idea de que tener un busto pequeño es una "enfermedad" que hay que "curar" en el quirófa no. Se recomienda la remodelación costal, más conocida como la extracción de costillas flotantes, para 
"mejorar el contorno corporal de una mujer que, por razones personales, piense que su cintura no está suficientemente marcada y quiera mejorar su apariencia y su autoestima"12. La alteración de la figura se describe como una "necesidad" y se compara con imágenes de personajes deHollywood. Seanuncian combos para el y para ella: "consulta, opérate y di sfrútal o en pareja", y se ofrecen "tratamientos" completos simul táneos con odontología cosmética ${ }^{13}$.

La medicina al servicio de la belleza y de la manipulación del cuerpo salió de los hospitales y entró rodeada de lujo al centro comercial. Para quéir a un hospital, Ileno de genteenferma, cuando ya se puede ir a una boutique donde se entra con un cuerpo "en mal estado" y se sale con el soñado, se recibe atención, cómodamente, por unas horas y se vive "el milagro" del bisturí14. El discurso utilizado y lo que ocurre en este campo clama por ser investigado.

Entre los procedimientos cosméticos se incluyen altas cirugías, implantes y el uso de sustancias inyectables, además de la toxina ya mencionada, como el colágeno, la silicona o la grasa de la mi sma paciente retirada de otro lugar del cuerpo. Las mujeres pobres de Colombia, por no quedarse atrás, han utilizado aceite de cocina o silicona industrial y se han practicado cirugías en sitios inadecuados, con consecuencias fatales ${ }^{15}$. La propaganda no habla de los riesgos de los implantes de silicona ni de las demandas que enfrentan las compañías fabricantes por los problemas asociados. Los implantes de silicona han resultado en una serie de problemas que incluyen rupturas, endurecimientos, incapacidad de lactar y de examinar el seno, dificultando la prevención del cáncer. Las revistas vienen atiborradas de fotos digitalizadas de mujeres con cuerpos "perfectos", ofreciendo servicios, como si fuera lo más sencillo y natural del mundo ${ }^{16}$. Es tal la proliferación deofertas en Colombiaque ya se ofrecen "paquetes turísticos" queincluyen transporte, al ojamiento y, desde luego, la cirugía y la terapia

2. http://www.cirugiaestetica.ws/contorno_corporal/ remodelacion_costal.htm

13 Para mayor detalle puede consultarse la revista $\mathrm{Cro-}$ mos de abril de 2004, número especial dedicado a la cirugía plástica. De igual manera, el periódico El Tiempo ha publicado especiales en conjunto con la Sociedad de Cirugía Plástica

14. "Shop, then drop in for surgery. Rodeo drive plastic surgery". Fortune Magazine. N oviembre de 2003

15 Una inyección de grasa en los glúteos llevó a la muerte a Erlin 0 spina, una caleña de veintiséis años, el 16 de enero del 2005

16. Ernesto Andrade V. "Cómo se hace una teta". Revista Soho. 51. 2004: 137. 
de recuperación a mujeres que vienen de otros países, a menores costos.

Aunque no se cuente con los recursos se hace un esfuerzo por reducir o aumentar el tamaño de los labios, I os senos, los glúteos, respingar la nariz, quitar arrugas y rellenar o vaciar muchas partes del cuerpo. Todo esto tiene un efecto negativo muy profundo. Los efectos colateral es de las cirugías incluyen infección, hemorragias, edemas, cicatrices, daño en los nervios, pérdida dela piel $y$, por supuesto, la muerte.

Las páginas brillantes y coloridas de las revistas dirigi das a las mujeres rara vez hablan de los riesgos o de las muertes resultado de estos procedimientos. En 2000 murieron en Neiva dos hermanas, menores de dieciocho años, durante cirugías cosmé ticas que les habían dado como regalo de cumpleaños ${ }^{17}$. Pocos

17. “La liposucción, entre la estética y la muerte". El Espectador. Sábado 29 de enero de 2000: 4A.

18. "Una víctima más de la lipoescultura". El Espectador. Sábado $\mathrm{D}$ de febrero de 2000: 9 A. meses después, en Valledupar faIlecieron la esposa del secretario de Educación del departamento del Cesar, y la jefe de impuestos de la gobernación ${ }^{18}$. En los primeros días de 2005 en Cali murió otra mujer, y el caso de la madre de la cantante Marbelle aún se recuerda, por mencionar sól o al gunos de los pocos que llegan a los medios.

Un reporte del Instituto de Medicina Legal y Ciencias Forenses (Giraldo, 2002) muestra el caso de una paciente que lleva exámenes previos a una li posucción con resultados aparentemente normales, quelediagnosticaban excel entes condiciones. Después de cuatro horas de la cirugía de liposucción, rinoplastia ambulatoria y plastia de mamas, presentó colapso cardi ovascular súbito. Fue llevada a la unidad de cuidados intensivos de una clínica, donde se le diagnosticó muerte cerebral. Los resul tados de la necropsia describen:

\footnotetext{
Sistema muscular presenta grandes hematomas en tejido muscular subcutáneo, donde se realizó el procedimiento de liposucción. Historia de intervención quirúrgica por estética, coagulación intravascular diseminada, conocida como coagulopatía de consumo desencadenada por la intervención deliposucción. La pacienteperdió 4.061 cc de sangre, es decir, el 97,6\% del volumen sanguíneo. Se sentencia al cirujano plástico y al anestesiólogo por homicidio culposo.
} 
La frecuencia de estos casos ha propiciado denuncias sobre las muchas irregularidades que existen en ese campo, pues hay quienes realizan cirugías estéticas sin la preparación ni el conocimiento necesario, en sitios inadecuados y sin condiciones mínimas de higiene ${ }^{19}$. Pero no por esto deja de haber toda clase de aberraciones, como las que fomentan realities como Extreme makeover, dondelas concursantes reciben la operación con la que han soñado. En una emisora nacional se creó un sorteo en el que las concursantes enviaban una fotografía señalando la parte del cuerpo que querían modificar, diciendo por qué querían cambiarla. El premi o era una cirugía estética. Los eventos de bel leza y el modelaje son otros en los que se patrocina el culto por el cuerpo, culto que tiene un lugar central en los noticieros y las propagandas dela televisión colombiana. Hay, incluso, concursos de belleza dondeel requisito principal es tener, ipor lo menos!, una cirugía plástica²0.

Es paradójico que el culto a la figura del gada con aparienciajoven se

19. Revista Ámbito Médico. Volumen 6(60). 0 ctubre de 2003 http:// www.medilegis.com. "Una cirugía estética no es como ir a la peluquería". El País. Cali. 16 de enero de 2005

20. http://www.univision.com/content/ content.jhtml? cid $=516878$ note más en países como Estados Unidos, donde la obesidad fue declarada como un problema de salud pública, lo que contrasta con la situación de países en los que aún se muere de hambre. La noción de peso ideal está atada a la de belleza y varía con el tiempo y el género; así, mientras en al gunos casos se espera la figura rellena, como en al gunas especialidades deportivas masculinas -como el sumo japonés o el fútbol americano-, en determinados momentos de la historia el sobrepeso en las mujeres se ha considerado una cual idad importante, necesaria parala lactanciay la reproducción o, en su defecto, cargada de erotismo.

Los desórdenes al imentarios producidos por las di etas extremas son cada vez más comunes entrelas jovencitas, al igual que las dependencias produci das por las anfetaminas y otras sustancias usadas en el control del peso. Muchas jóvenes comienzan a fumar para reducir el apetito y controlar la irritabilidad queles produce la sensación constante de hambre.

Surgen muchas preguntas como materia de investigación: ¿dado el al to costo de alcanzar este ideal, por qué las mujeres siguen intentándolo? ¿Por quése está convirtiendo en la norma? ¿Por qué la medicina y las mujeres mismas participamos en esta fetichización del cuerpo, que además define lo femenino en términos de 
sumisión? ¿Será, como dice Morgan (2003), que para que una mujer sea amada, contratada, el egida o, simplemente, pueda vivir, debe someterse a la estética y al bisturí del cirujano?

Todo esto nos Ileva a reflexionar acerca de cómo las ideas sobre el cuerpo, la bel leza y la enfermedad deben estar ligadas a agendas políticas y sociales. La medicina y la tecnología se ponen al servicio de quienes, por diferentes motivos, entre ell os el racismo, quieren alterar su imagen corporal disminuyendo los rasgos que acentúan ciertas características fenotípicas, y que van más allá de usar lentes de contacto sól o para aclarar el color delos ojos, como en el caso de orientales que quieren modificar la forma de los ojos o de personas de origen afro que quieren cambiar la forma de la nariz o alisarse el pelo (Kaw, 2003).

Para cerrar la exposición sobrela cirugía plástica hago mención a otros aspectos, muy interesantes también, que se resuel ven en el quirófano y que afectan las relaciones y jerarquías de género, como son los cambios de sexo y al teración de características corporales con la intención de modificar la identidad de género.

\section{LAS DIFERENTES VIOLENCIAS \\ Y SUS EFECTOS EN LA SALUD DE LAS MUJERES}

D

E LA VIOLENCIA DEL BISTURí PERVERSO PASAMOS A OTROS TIPOS DE violencia y a su efecto en la salud y el cuerpo de las mujeres. Por razones de espacio no me detendré demasiado, sino que melimitaréa resal tar al gunos puntos principales. El trauma físico y psicológico resultante de la violencia de género, que ocurre en

21 El Instituto de Medicina Legal registró ciento cuarenta y siete homicidios por violencia doméstica, es decir, un homicidio cada tres días; y se emitieron 36511dictámenes a mujeres víctimas del abuso conyugal. Instituto de Medicina Legal y Ciencias Forenses. 1999. Lesiones de causa externa, Colombia 1998 la casa y a puerta cerrada, es un grave problema desal ud pública en muchos países, incluido Colombia, en dondela gran mayoría de víctimas (92\%) son mujeres ${ }^{21}$.

El sistema médico tampoco está preparado para apoyar a las víctimas. En los mejores casos se limita a atender el trauma físico, sin que las afectadas puedan expresar sus preocupaciones y miedos y regresen, sin alternativas, a sus casas a enfrentar la misma situación. Es decir que, de nuevo, la mujer desaparece detrás del trauma. La intervención y el apoyo del estado son 
deficientes y los pocos programas creados con el objetivo de fortal ecer la familia han si do víctima de recortes presupuestal es.

Por otra parte, la violencia de los conflictos armados afecta también a las mujeres, de diversas maneras. La violación y la agresión sexual es un arma de guerra (Rojnik, Andolsek-Jeras y Obersnel-Kveder, 1995) y tiene entre sus consecuencia embarazos no deseados, enfermedades de transmisión sexual y secuelas emocionales, problemas que apenas comienzan a reconocerse en Colombia (Fiscó, 2004; IIsa, 2003). Las mujeres, quienes son la mayoría de desplazadas y refugiadas en el mundo, tienen necesidades especiales en términos de salud y son las que menos disponen de acceso a los servicios de salud. Esto ha hecho que entidades como Amnistía Internacional (1992), y las Naciones Unidas (Consortium for Health and Human Rights, 1998) diseñen propuestas relacionadas con estos problemas. En el caso de A mérica latina se ha llamado la atención sobre las mujeres y la tortura, incluyendo el maltrato doméstico (Bunster, 1997). Por lo general, la salud de las mujeres en los conflictos no se encuentra en las agendas estatales (Heise, 1993 Herman, 1992; Yamim, 1997), a pesar de problemas adicional es como las lesiones dejadas por las minas antipersona, las mutilaciones, los resultados devastadores de los combates, las heri das dejadas por bombas y muchas otras secuelas. La mayor parte de los muertos en combate son hombres, por lo que debe tenerse en cuenta el impacto psicol ógi co en las mujeres que sobreviven a sus compañeros, en los huérfanos y en otros familiares. Por último, es necesario mencionar el problema global del tráfico de mujeres (Skrobanek et al., 1997), la prostitución y el aborto forzados.

\section{CONCLUSIÓN}

D ESPUÉS DE ESTE RECORRIDO POR ALGUNOS DE LOS ASPECTOS RELACIOnados con el cuerpo, la violencia, la subordinación y la sal ud de las mujeres, se plantean varios interrogantes, muchos de ellos relacionados con los derechos humanos. Seolvida muchas veces que cuando se habla de salud reproductiva, de la cirugía plástica o del impacto de la violencia, deben tenerse presentes los derechos de las mujeres y el lugar en donde se encuentran situadas. Para esto es necesario un cuestionamiento desde la 
antropol ogía médica, haciendo claras las intersecciones entreel género, la clase social y, en nuestro caso, con los problemas de salud específicos de las poblaciones indígenas y afro y su relación con la seguridad social (Ayora, 1995) y el conflicto armado.

A pesar de que la biomedicina ha solucionado muchos problemas milenarios de salud, ha creado también nuevos problemas y dilemas éticos. El control que tiene sobre procesos como el parto ha traído beneficios, pero ha hecho a las mujeres dependientes por completo de la tecnología, con pocas opciones de cuestionar la imposición de la autoridad, dando menos importancia a la dimensión humana, psi cológica o social.

No pueden dejar de mencionarse otros problemas de sal ud en relación con el género, que por razones de espacio no se desarroIlaron en este documento, como son los que ocurren en el área de trabajo. En Colombia, por ejemplo, se ha llamado la atención sobre el impacto delas fumigaciones para erradicar cultivos de uso ilícito y sobrelas enfermedades características de las mujeres que trabajan en la industria de las flores (Medrano, 1982), pero poco se habla de los problemas de salud comunes a las mujeres en el hogar, como las quemaduras y cortadas, o de otros accidentes domésticos. Se llama la atención a otro asunto de importancia global, que quedó apenas mencionado en este artículo, el VIH/ sida, que por fortuna ya comenzó a trabajarse en nuestro medio desde la perspectiva de género y la antropol ogía médica (Ávila, 1996; García, Fadul y Castellanos, 1995).

En Col ombia se encuentran, coexisten, interactúan y chocan diferentes sistemas de sal ud con otros factores que contribuyen a la producción social de la mala salud, como, por ejemplo, el conflicto armado, la pobreza, las ideologías de superioridad de género y las políticas estatales que dejan de lado la prevención y la investigación, que cierran hospital es y promueven la atención de la salud desde entidades privadas con ánimo de lucro. Dentro de esto actúan hombres y mujeres, con procesos históricos y cultural es diferentes que merecen una mejor atención en la salud y una mayor calidad de vida. 


\section{BIBLIOGRAFÍA}

Alcaraz, G. et al. 1993 "Aspectos culturales en torno a la calidad de vida de las gestantes embera". En C. Pinzón, R. Suárez y G. Garay (comps). Cultura y salud en la construcción de las Américas: políticas y experiencias. Ican- Colcultura. Bogotá.

Amnesty InTERnATIONAL. 1992. Ethical codes and declarations relevant to the health professions. $3 r d$ edition. Amnesty International. Londres.

Arango, L. G. 1992. Estatus adolescente y valores asociados con la maternidad y la sexualidad en sectores populares urbanos de Bogotá. En A. C. Defossez, D. Fassin y M. Viveros (eds.). IFEAUniversidad Externado de Colombia. Bogotá.

ÁviLA S., E. 1996. “Factores sociol culturales asociados a la transmisión de VIH". Ican. Bogotá.

Ayora Álvarez, S. 1995 La seguridad social integral y las comunidades indígenas coama. Bogotá. Fundación Gaia.

BaumslaG, N. y D. L. Michels. 1995 "The global search for formula sales". En Milk, money, and madness: the culture and politics of breastfeeding. Bergin and Garvey. Westport.

BAYLEY, P. et al. 1988 "Estudio hospital ario del aborto ilegal en Bolivia”. Boletín de la Oficina Sanitaria Panamericana. OPS. Washington.

Browner, C. H., NAnCy Ann. 1995 "The normalization of prenatal diagnostic screening". En Faye Ginsberg y Rayna Rapp (eds.). Conceiving the new world order: The global politics of reproduction. University of California Press. Berkeley.

Buckley, T. y A. GotTlieb. 1988. "A critical appraisal of theories of menstrual symbolism". En T. Buckley y A. Gottlieb (eds.). Blood magic. University of California Press. Berkeley.

BUNSTER B., X. 1997. "Surviving beyond fear: Women and torture in Latin America". En Miranda Davies (ed.). Women and violence. Zed Books. Londres.

Castaneda, X., D. L. Billings, J. Blanco. 2003 "Abortion beliefs and practices among midwives (Parteras) in a rural Mexican township". Women \& Health. Old Westbury. 37 (2).

Consortium for Health and Human Rights. 1998. "Health and human rights: A call to action on the 50th anniversary of the Universal Declaration of Human Rights". Journal of the American Medical Association. 280 (5). 
Cosminsky, S. 1977. “El papel de la comadrona en Mesoamérica”. En América Indígena. 37 (2).

DAVIS, D. 1996. "The cultural constructions of the premenstrual and menopause syndromes". En C. Sargent y C. Brettell. Gender and health: An international prespective. Prentice Hall. New Jersey.

Douglas, M. 1977. Pureza y peligro: un análisis de los conceptos de contaminación y tabú. Siglo XXI. Madrid.

DoyAL, L. 1995 What makes women sick: Gender and the political economy of health. Rutgers University Press. New Jersey.

ECheVerRI, L. 1990. Familia y vejez en Colombia. Perspectivas año 2000. Universidad Nacional de Colombia-Colciencias. Bogotá.

FIscó, S. 2004. “La violencia sexual contra la mujer en el marco del conflicto armado colombiano: una aproximación desde la investigación para la paz y la perspectiva de género". Trabajo de grado. Departamento de ciencia política, facultad de ciencia política y relaciones internacionales. Pontificia Universi dad Javeriana. Bogotá.

GálveZ, A. et al. 2002. El mañana que ya entró. La fecundidad en los pueblos indígenas de Antioquia. Universidad deAntioquia. Medellín.

García S., C. (coord.). 2003 Estado del arte sobre la situación de las mujeres. Bogotá 1990-2002. Departamento Administrativo de Protección Social-Universidad Central. Bogotá.

García, R., R. Fadul y C. Castellanos. 1995 “Manejo de las diferencias de género en educación y consejería sobre VIH-sida". Salud. Culturas de Colombia. 15

Giraldo, C. A. (coord.). 2002. "Cirugía estética y muerte intraanestésica. Correlación clínica y medicolegal". Casos Forenses. 15 Instituto Nacional de Medicina Legal y Ciencias Forenses. Bogotá.

GoRDON, D. 1991 "Female circumcision and genital operation in Egypt and the Sudan: A dilemma for medical anthropology". Medical Anthropology Quarterly. 5

Guevara, R. D. 1997. La mujer inga: proyección histórica, genérica y de identidad cultural. Proprensa Difundir. Bogotá.

Gutiérrez de Pineda, V. y R. Pineda G. 1985 “Ciclo vital y chamanismo entre los indios del Chocó". Revista Colombiana de Antropología. 25

GutiérRez, Y. y R. López. 1999. “Estatus femenino ¿atado al cordón umbilical? El comportamiento reproductivo entre los embera y zenú de Antioquia, Colombia". En M. Viveros y G. Garay (comps.). Cuerpo, diferencias y desigualdades. Universidad Nacional de Colombia-CES. Bogotá.

Haraway, D. 1991 Ciencia cyborgs y mujeres. Cátedra. Madrid. 
HeISE, L. 1993 “Viol ence against women: The missing agenda”. En M. Koblinsky, J. Timyan y J. Gay (eds.).The health of women. A global perspective.

Herman, J. 1992. Trauma and recovery. Basic Books. Nueva York.

HeRnÁndeZ, I. 1997. "Salud reproductiva y planificación familiar o salud reproductiva versus planificación familiar: el concepto de salud reproductiva y el fondo de población de las Naciones Unidas: una perspectiva institucional". Ponencia en el IV Congreso latinoamericano de ciencias sociales y medicina, Cocoyoc, México.

Hubbard, R. 1985 "Prenatal diagnosis and eugenic ideology". Women's Studies. 8 (6).

ILSA. 2003 Mujer y conflicto armado. Tercer Informe sobre violencia sociopolítica contra mujeres, jóvenes y niñas en Colombia 2002. Instituto Latinoamericano deServicios Legales Alternativos, Ilsa. Bogotá.

Kaw, E. 2003 "Medicalization of racial features: Asian-American women and cosmetic surgery". En Rose Weitz (ed.).The politics of women's bodies. Oxford University Press.

KelLeR, E. F. 1985 Reflexiones sobre género y ciencia. Edicions Alfons el Magnanim, Generalitat Valenciana.

Krieger, N. y E. Fee. 1996. “Man-made medicine and women's health. The biopolitics of sex/gender and race/ethnicity". En Kary L. Moss (ed.). Man-made medicine; women's health, public policy, and reform. Duke University Press. Durham.

Leal Garza, C. H. et al. 1995 “Estudio citogenético en parejas con aborto habitual". Antropología biológica. Asociación Latinoamericana de Antropología Biológica. Bogotá.

LI, D. 1992. "Barreras que afectan el cuidado de la salud reproductiva en las áreas marginales del Perú". Seminario mujeres de los Andes. Condiciones de vida y salud (Quito, 199Д. Ifea. Bogotá.

Lindenbaum, S. 1979. Kuru sorcery: Disease and danger in the New Guinea highlands. Mayfield Publishing Company. Mountain View.

LóPEZ R., T. 1945 “Algunos datos sobre la menstruación, la preñez, el aborto y el parto entre los indios venezolanos". Acta Venezolana. Boletín del Grupo Caracas de la Sociedad Interamericana de Antropología y Geografía. 1

LoRber, J. 1997. Gender and the social construction of illness. Sage Publications.

MARTIN, E. 1987. The woman in the body: A cultural analysis of reproduction. Beacon Press. Boston. 
1990. "Science and women's bodies: Forms of anthropological knowledge". En M. Jacobus, E. Fox Keller y S. Shuttleworth (eds.). Body politics: Women and the discourses of science. Routl edge. Nueva York.

1996. "The egg and the sperm: How science has constructed a romance based on stereotypical male-female roles". En C. Sargent y C. Brettell. Gender and health: An international perspective. Prentice Hall. New Jersey.

McKee, L. A. 1982. "Los cuerpos tiernos: simbolismo y magia en las prácticas post-parto en Ecuador". América Indígena. 42 (4).

MedRano, D. 1982. “Desarrollo y explotación de la mujer: efectos de la prol etarización femenina en la agroindustria de fl ores en la Sabana de Bogotá". En Magdalena León (ed.). Debate sobre la mujer en América Latina y el Caribe. Volumen I. La realidad colombiana. Asociación Colombiana para el Estudio de la Población. Bogotá.

Merchant, C. 1989. Death of nature: Women, ecology, and the scientific revolution. Harper and Row. San Francisco.

Merchant, K. y K. M. Kurz. 1993 “Women's nutrition through the life cycle: Social and biological vulnerabilities". En M. Koblinsky, J. Timyan y J. Gay (eds.). The health of women: A global perspective. Westview Press. Boulder.

MILLER, B. 1997. "Female infanticide and child neglect in rural north India". En C. Brettell y C. Sargent (eds.). Gender in cross-cultural perspective. Prentice Hall. Englewood Cliffs.

Morgan, K. P. 2003 "Women and the knife: Cosmetic surgery and the colonization of women's bodies". En The politics of women's bodies. Oxford University Press. Nueva York.

Ortiz R., F. y E. Pradilla. 1987. "Indígenas de los Ilanos orientales". En Introducción a la Colombia amerindia. ICAN. Bogotá.

Petchesky, R. y K. Judd (eds.). 1998. Negotiating reproductive rights: Women's perspectives across countries and cultures. Zed Books. Londres.

PuYANA, Y. 1985 "El descenso de la fecundidad por estratos sociales". En E. Bonilla (comp.). Mujer y familia en Colombia. Plaza y Janés. Bogotá.

Ramírez, N. 1983 "Asistencia clínica de la maternidad en Bogotá". Monografía de grado. Departamento de antropología, facultad de ciencias humanas. Universidad Nacional de Colombia. Bogotá.

RAPP, R. 1990. "Constructing amniocentesis: Maternal and medical discourses". En F. Ginsberg y A. Lowenhaupt Tsing (eds.). Uncertain terms: Negotiating gender in American culture. Beacon Press. Nueva York. 
Rico DE Alonso, A. 1986. Madres solteras adolescentes. Universidad Javeriana y Plaza y Janés. Bogotá.

Rojnik, B., L. Andolsek-Jeras y D. Obersnel-Kveder. 1995 "Women in difficult circumstances: War victims and refugees". International Journal of Gynecology and Obstetrics. 48.

Ruzek, S., V. Olesen y A. E. Clarke (eds.). 1997 Women's health; complexities and differences. Ohio State University Press. Columbus.

Saltzberg, E. y J. Chrisler 1995 "Beauty is the beast: Psychological effects of the pursuit of the perfect femal e body". En J. Freeman (ed.). Women: A feminist perspective. Mayfield Publishing. Mountain View.

Sargent C. y C. Brettell. 1996. Gender and health: An international perspective. Prentice Hall. Nueva Jersey.

Schaller, J. G. y E. O. Nightingale. 1992. "Children and childhood's: Hidden casualties of war and civil unrest". JAMA. 268 (5).

SCHePER-Hughes, N. 2002. "Culture, scarcity, and maternal thinking: Maternal attachment and infant survival in a Brazilian shantytown". En Margaret Spinelli (ed.). Infanticide, psychosocial and legal perspectives on mothers who kill. American Psychiatric Press. Nueva York.

SCHIEBINGER, L. 2004. ¿Tiene sexo la mente? Las mujeres en los orígenes de la ciencia moderna. Grupo Anaya. Madrid.

Sevilla C., Elías. 2003 El espejo roto. Editorial Universidad del Valle. Cali.

SkrobaneCK, S. et al. 1997. El tráfico de mujeres: realidades humanas en el negocio internacional del sexo. Narcea. Madrid.

SonTAG, S. 1989. El sida y sus metáforas. Muchink Editores. Madrid.

TABARES, E. 1995 “Mortalidad y subsistencia, la lucha de un pueblo indígena”. Salud, Culturas de Colombia. Sida, Mujer y Género. 15

Tovar, P. 2004. “De historias, histerias e histerectomías: Ia construcción de los discursos médicos y los imaginarios sobre la reproducción femenina". En M. Viveros (coord.). Memorias seminario-taller: hacia una agenda sobre sexualidad y derechos humanos en Colombia. Grupo de estudios en género, salud y sexual idad en América Latina (GESSAM), CES-Universidad Nacional de Colombia. Bogotá.

URIBE, R. 2002. La invención de la mujer: nacimiento de una escuela médica. Fondo de Cultura Económica. México.

URREA, F. et al. 2004. "Sexualidade e saúde reprodutiva em jovens negros, homens e mulheres de setores populares em Cali". En S. Monteriro y L. Sansone (orgs.). Etnicidade na América Latina: um debate sobre raça, saúde e direitos reprodutivos. Fiocruz Editora. Rio de Janeiro. 
Vargas, L. A., E. Matos M. 1973 “El embarazo y el parto en el México prehispánico". Anales de Antropología. 10.

ViVEROS, M. 1997. "El aborto en Colombia: debate público y dimensiones sociocultural es, 1975 1994". Ponencia preparada para Latin American Studies Association, Guadalajara, México. www.abortolegal.org/ abortoColombia.pdf

Viveros, M. y F. Gómez. 1998. "La el ección de esterilización masculina”. En Mujeres, hombres y cambio social. Universidad Nacional de Colombia. Bogotá.

WARTENBERG, L. 1999. Embarazo precoz y aborto adolescente en Colombia. Universidad Externado de Colombia. CIDS. FNUAP. Bogotá.

WARTEnBerg, L., H. SalCedo. 1997. "La identidad de género en la toma de decisión del aborto inducido". Informes antropológicos. 9.

Yamin, A. 1997. “Transformative combinations: Women's health and human rights". J. of the American Medical Women's Association. 52 (4).

Zamudio, L., N. Rubiano y L. Wartenberg. 1992. El aborto en Colombia: características demográficas y socioculturales. Universidad Externado de Colombia. Bogotá. 